\title{
谱编码衍射干涉产生相位体的 可视像和特征识别
}

\author{
刘立人 \\ （中国科学院上海光学精密机械研究所）
}

大多数生物和医学标本属于相位物体, 用普通显微镜不能直接观察, 需要染色, 这对活细 胞有害. 相位反祄、中央暗场和纹影等法能使相位体转换成目视可见 ${ }^{[1]}$. 这些方法在物理、化 学和工业上也有着重要的应用。但相衬法等并不具备更多的信息处理性质.上述应用中常需 要对相位体进行特征识别. 因此同时实现相位体的可视观察和特征识别是很有意义的, 将具 有较大的应用价值.

光学上实现特征识别最著名的是全息匹配滤波器法 ${ }^{[2]}$. 但是它不能直接用于相位体, 先 要把相位体转变为可视图像. 一种方法仍是对样品染色 ${ }^{[3]}$; 另一种是用相祄显微镜取得可视 照片 ${ }^{[4]}$. 或者直接作为实时输入 ${ }^{[5]}$. 但匹配滤波进行特征相关识别时完全不能同时对物体成 像。

我们已提出过用编码光栅滤波或相关的方法来产生相位体的等位线条纹 ${ }^{[6,7]}$. 对于弱变 化相位体干涉形式取决于编码光栅的傅里叶谱分布 ${ }^{[8]}$. 这能设计各种形状的编码光㮽以取得 不同含义的干涉像. 然而像点的光场与编码光㫼的傅里叶谱和物体的相关积分有关，这意味 着可以进行相位特征识别. 本文报道这种同时实现相位体的可视观察和特征识别的新原理.

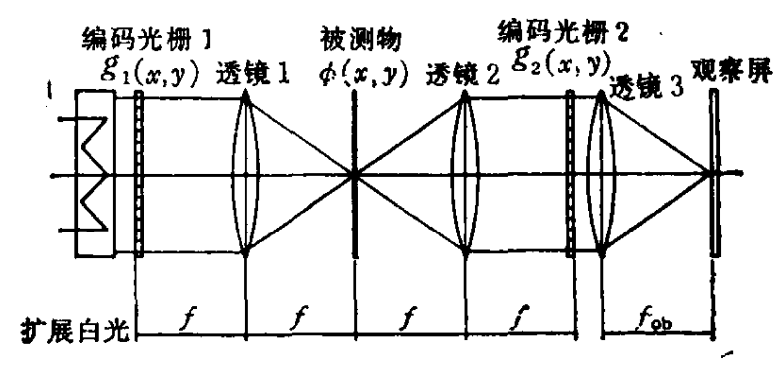

图 1 典型的谱行射干涉装置

\section{一、编码楖谱衍射千涉原理}

典型系统见图 1. 扩展光照明. 编码光栅为 $g_{1}$ 和 $g_{2}$, 相应谱函数为 $G_{1}\left(\frac{x}{\lambda f}, \frac{y}{\lambda f}\right)$ 和 $G_{2}$ $\left(\frac{x}{\lambda f}, \frac{y}{\lambda f}\right)$. 透镜 1 和 2 用作傅里叶变换. 物体经透镜 2 和 3 成像于观察屏上,放大率为 $M=$ $f_{\mathrm{ob}} / f$. 为考虑特征识别, 下面简引文献 $[8]$ 的结果并加以扩充.

本文 1985 年 12 月 10 日收到. 1986 年 8 月 3 日收到修改稿. 
观察屏上任一像点的场强为相关积分:

$$
E(x, y ; \lambda)=\int_{-\infty}^{\infty} \int_{0} G_{1}\left(\frac{\alpha}{\lambda f}, \frac{\beta}{\lambda f}\right) G_{2}\left(\frac{\alpha}{\lambda f}, \frac{\beta}{\lambda f}\right) e^{i \phi\left(\alpha-\frac{x}{M}, \beta-\frac{y}{M}\right)} d \alpha d \beta,
$$

其中 $\phi(x, y)$ 表示相位体的相位分布. 相应像点的亮度为 $|E|^{2}$. 记作乘积谱 $G_{s}=G_{1} G_{2}$, 则像点亮度与乘积谱经受物体相位延迟后合成干涉强度成正比，从而实现了相位体的可视观 察. 白光照明下像点亮度为对整个光源的可视波长积分. 编码光栅的色散衍射将使干涉像出. 现彩色。

无相位变化时的背景场强为

$$
\begin{aligned}
E_{b}(\lambda) & =\int_{-\infty}^{\infty} \int_{1} G_{1}\left(\frac{\alpha}{\lambda f}, \frac{\beta}{\lambda f}\right) G_{2}\left(\frac{\alpha}{\lambda f}, \frac{\beta}{\lambda f}\right) d \alpha d \zeta=\left.G_{1}\left(\frac{x}{\lambda f}, \frac{y}{\lambda f}\right) \otimes \otimes G_{2}\left(\frac{-x}{\lambda f}, \frac{-y}{\lambda f}\right)\right|_{x=y=0} \\
& =\left.G_{1}\left(\frac{x}{\lambda f}, \frac{y}{\lambda f}\right) * * G_{2}\left(\frac{x}{\lambda f}, \frac{y}{\lambda f}\right)\right|_{x=y=0},
\end{aligned}
$$

这里 $\otimes \otimes$ 和**分别代表两维卷积和相关积分.

由于制作方便, 最常用的振幅型编码光栅是取值 0 和 1 的双元实函数. 它具有如下性 质: $g(x, y)=g(x, y) g(x, y)$, 所以 $G\left(f_{x}, f_{y}\right)=G\left(f_{x}, f_{y}\right) \otimes \otimes\left(f_{x}, f_{y}\right)$, 以及 $G\left(f_{x}, f_{y}\right)=$ $G^{*}\left(-f_{x},-f_{y}\right)$. 使用上又常使两编码栅有相同的函数以取得较强的乘积谱衍射. 这时可能 的编码柟组合有: (1) 两编码栅相互成像, 即 $g_{1}(x, y)=g_{2}(-x,-y)=g(x, y)$, 因此 $G_{s}=|G|^{2}, E_{b}=G(0,0)$. (2) 反成像, 即 $g_{1}(x, y)=g_{2}(x, y)=g(x, y)$, 因此 $G_{s}=G^{2}$, $E_{b}=\left.G * * G\right|_{f_{x}=f_{y}=0}$. (3) 互补成像, $g_{1}(x, y)=g(x, y), g_{2}(x, y)=1-g(-x,-y)$, 因: 此 $G_{s}=G(0,0)-|G|^{2}, E_{b}=0$. (4) 互补反成像, $g_{1}(x, y)=g(x, y), g_{2}(x, y)=1-$ $\mathrm{g}(x, y)$, 因此 $G_{s}=G(0,0)-G^{2}, E_{b}=G(0,0)-\left.G * G\right|_{f_{x}=f_{y}=0}$. 可见成像组合时乘积 谱为实数,互补成像时产生暗背景.

另一种常用的编码栅是双值 $(0, a)$ 纯相位型, 这可简单地由双元光栅 $g(x, y)$ 漂白而 得, 即 $g_{\phi}(x, y)=e^{j a g(x, y)}=1+A g(x, y)$, 而 $A=\cos a-1+j \sin a$. 同样; (1) 两编码 栅成像, 则 $G s=\delta+2 A G(0,0)+A^{2}|G|^{2}, E_{b}=1+\left(A^{2}+2 A\right) G(0,0)$. (2) 反成像, 则 $G_{s}=\delta+2 A G(0,0)+(A G)^{2}, E_{b}=1+2 A G(0,0)+\left.A^{2} G * G\right|_{f_{x}=f_{y}=0}$. (3) 互补成像, 则 $G_{s}=e^{j a}\left[\delta+2(\cos a-1) G(0,0)+2(1-\cos a)|G|^{2}\right], E_{b}=e^{j a}$. (4) 互补反成像, 则

$$
\begin{gathered}
G_{s}=\epsilon^{j a}\left[\delta+2(\cos a-1) G(0,0)+2(1-\cos a) G^{2}\right], \\
E_{b}=e^{j a}\left[1+2(\cos a-1) G(0,0)+\left.2(1-\cos a) G * * G\right|_{f_{x=f y}=0}\right] .
\end{gathered}
$$

当 $a=\pi$ 以及编码桶开口为周期之半时将消除乘积谱中的直流分量, 这通常是编码柟原设计 中并不存在的 ${ }^{[9]}$. 正成像下当 $a=\frac{\pi}{2}$ 时 $E_{b}=0$, 为暗场观察. 互补成像下永为亮场观察.

\section{二、特 征 识 别}

特征识别表现为观察屏上出现一个近似 $\delta$ 函数的相关点. 可以看出背景亮度有影响, 因 此需要加入参考光对相关点进行干涉调制, 便之明显区别于背景. 由此应设计编码棚的乘积 谱由特征函数和参考光两部分组成, 即

$$
G_{s}\left(f_{x}, f_{y}\right)=G_{c}\left(f_{x}, f_{y}\right)+G_{r}\left(f_{x}, f_{y}\right),
$$

则像点场强为特征场强 $E_{c}$ 和参考场强 $E_{r}$ 之和: 


$$
\begin{aligned}
& E_{c}(x, y ; \lambda)=\iint_{-\infty}^{\infty} G_{c}\left(\frac{\alpha}{\lambda f}, \frac{\beta}{\lambda f}\right) e^{i \phi\left(\alpha-\frac{x}{M}, \beta-\frac{y}{M}\right)} d \alpha d \beta, \\
& E_{r}(x, y ; \lambda)=\iint_{-\infty}^{\infty} G_{r}\left(\frac{\alpha}{\lambda f}, \frac{\beta}{\lambda f}\right) e^{j \phi\left(\alpha-\frac{x}{M}, \beta-\frac{y}{M}\right)} d a d \beta .
\end{aligned}
$$

首先考虑只识别同相位分布的花样, 这时设计 $G_{c}$ 为两维双元函数. 如果相应某像点 $\left(x^{\prime}, y^{\prime}\right)$ 的物体的同相位分布花样与特征函数 $G_{c}$ 相同, 那么像点上的场强将达到最大:

$$
E_{c M}\left(x^{\prime}, y^{\prime} ; \lambda\right)=e^{i \phi_{0}} \int_{-\infty}^{\infty} \int_{c} G_{c}\left(\frac{\alpha}{\lambda f}, \frac{\beta}{\lambda f}\right) d \alpha d \beta=e^{i \phi_{0} E_{b c}(\lambda),}
$$

$\phi_{0}$ 为特征函数和物体的相移之和. 非重合位置上特征函数通过物体产生的场强不是同相垔 加,将远小于 $E_{c M}$ ，因此式 (6) 表示相关点峰值.

最简单的参考光可取干涉谱中的直流分量, 这是不和特征函数重叠的点函数, 显然

$$
E_{r}\left(x^{\prime}, y^{\prime} ; \lambda\right)=e^{i\left[\phi\left(-\frac{x^{\prime}}{M} \cdot-\frac{y^{\prime}}{M}\right)+\phi_{c r}\right]} \int_{-\infty}^{\infty} \int_{r}\left(\frac{\alpha}{\lambda f}, \frac{\beta}{\lambda f}\right) d \alpha d \beta=e^{i\left[\phi\left(-\frac{x^{\prime}}{M},-\frac{y^{\prime}}{M}\right)+\phi_{c r}\right]} E_{b r}(\lambda),
$$

$E_{b c}$ 和 $E_{b r}$ 为特征函数和参考光单独产生的背景场强. $\phi_{c r}$ 为特征函数与参考光之间的相 移. 或者设计参考光为同相两维分布函数并远离特征函数, 它不经过物体但人为地附加相位 移 $\phi_{a}$, 即

$$
E_{r}\left(x^{\prime}, y^{\prime} ; \lambda\right)=e^{j\left(\phi_{a}+\phi_{c r}\right)} E_{b r}(\lambda) .
$$

上述两种情况下, 显然相关点场强为干涉和

$$
E_{p}\left(x^{\prime}, y^{\prime} ; \lambda\right)=e^{i \phi_{0}}\left[E_{b c}(\lambda)+\left(e^{i \Delta \phi}-1\right) E_{b r}(\lambda)\right],
$$

这里

$$
\Delta \phi=\phi\left(-\frac{x^{\prime}}{M}, \frac{y^{\prime}}{M}\right)+\phi_{c r}-\phi_{0}
$$

或 $\Delta \phi=\phi_{a}+\phi_{c r}-\phi_{0}$. 在亮场观察下为特出特征识别相关点, 应使它为暗点. 这要求控制 条件 $\Delta \phi \approx \pi$ 和 $E_{b r} \approx E_{b c}$. 暗场观察下, 应使相关点为亮点, 因 $E_{b}=0$ 同样需使 $\Delta \phi \approx$ $\pi$.

为同时识别相位分布的花样和相位变化， $G_{c}$ 设计为振幅和相位都变化的两维复函数. 显 然,当

$$
e^{j \phi\left(\alpha-\frac{x^{\prime}}{M}, \beta-\frac{y^{\prime}}{M}\right)}=G_{c}^{*}\left(\frac{\alpha}{\lambda f}, \frac{\beta}{\lambda f}\right)
$$

时出现相关峰值:

$$
E_{c M}\left(x^{\prime}, y^{\prime} ; \lambda\right)=e^{j \phi_{0}} \int_{-\infty}^{\infty} \int_{-\infty}\left|G_{c}\left(\frac{\alpha}{\lambda f}, \frac{\beta}{\lambda f}\right)\right|^{\beta} d \alpha d \beta=B e^{j \phi_{0}},
$$

这里 $B$ 为正实数， $\phi_{0}$ 为特征函数和物体间可能存在的均匀相移差. 相关点干涉场强为

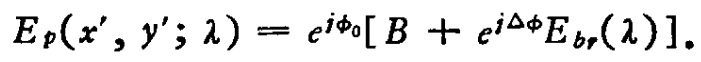

同样,亮场下应使 $\Delta \phi \approx \pi$, 暗场下应使 $\Delta \phi \approx 0,2 \pi$.

在特征函数和参考光的共同作用下相位体也产生可视像,千涉形式与所用的乘积谱有关. 


\section{三、实嬐}

设侍识别的特征相位分布是等相位圆环，图 2a 是样品图案，样品用漂白全息板制成，纯相 位型. 圆环形衍射可由圆光柱产生 ${ }^{[8]}$, 同时还存在中心直流项, 现用作参考光. 图 1 系统参数 为 $f-f_{o b}=135 \mathrm{~mm}$, 两圆光栅频率均为 5 条 $/ \mathrm{mm}$, 行射圆环半径为 $\lambda f / D$. 平均波长 $5000 \AA$ 上 $r_{\mathrm{avr}}=0.371 \mathrm{~mm}$, 最大可视波长 $7000 \AA$ 上. $r_{\mathrm{max}}=0.473 \mathrm{~mm}$, 最小可视波长 $4000 \AA$ 上 $r_{\mathrm{min}}=0.270 \mathrm{~mm}$. 图 $2 \mathrm{~b}$ 为观察屏上的像, 相位型图案已成为可视像. 其中三个圆环的宽度在 $r_{\max }$ 和 $r_{\min }$ 之间,出现了特征识别的中心相关点，与设想一致．全息板样品浸水涨泡以控制 $\Delta \phi \approx \pi$. 此时相关点和像都是黑条纹. 由于圆环的自相关性质, 成像环内外还会出现较浅的 圆环。

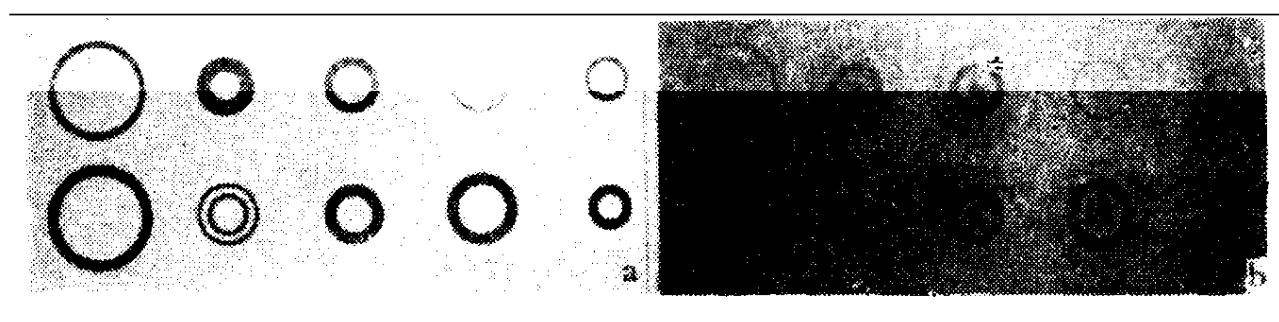

图 2

a 相位样品图案示意; b 样品成像图

本文详细讨论了系统的分析特性, 而对任一所需的相位特征识别则要求能合成出相应的 编码光柾,这可采用各种全息图的方法 ${ }^{[9-11]}$.

[1] Goodman, J. W., Introduction to Fourier Optics, McGraw-Hill, San Francisco, 1968, 144-146.

[ 2] ibid, $171-184$.

[ 3 ] Bond. R. L.. Mazumder, M. K., Testerman, M. K., Hsien, D., Science, 149(1973), 571-573.

[ 4 ] Almeida, S. P., Eu, J. K. T., Appl. Opt, 15(1976), 510-515.

[5] Partin, J. K., Almeida, S. P., Fujii, H., Holography in Medicine and Biology (Ed. Bally, G.), Springer-Verlag, Berlin, Heidelberg, New Ycrk, 1979, 73-76.

[6] Liu Liren, Appl. Opt., 21(1982), 2817-2826.

[ 7 ] Liu Liren, ibid., 22(1983), 3016-3024.

[8] 刘立人, 光学学报, 4(1984), 970-978.

[9] 刘立人、黄洪欣、王之江, 光学学报, 6(1986),988-996.

[10] Liu Liren, Acta Polytech. Seadinavica, 1985, 149: 325-328.

[11] 刘立人, 中国㴊光, 12(1985), 641-647. 